\title{
Metacognição: Um Apoio ao Processo de Aprendizagem
}

\author{
Célia Ribeiro ${ }^{1}$ \\ Universidade Católica Portuguesa
}

\begin{abstract}
Resumo
O presente artigo, a partir de uma incursão pela literatura, visa contribuir para a clarificação do conceito de metacognição. Neste sentido, procura evidenciar o papel das estratégias metacognitivas na potencialização da aprendizagem, ou seja, no desenvolvimento pelo aluno de modos eficazes para lidar com a informação proveniente do meio e com os próprios processos de pensamento. Argumenta-se que apesar da polêmica acerca do conceito de metacognição, é inegável a sua contribuição para a aprendizagem, uma vez que os treinos que contemplam atividades metacognitivas têm produzido melhores resultados no que se refere a realização escolar.

Palavras-chave: Metacognição; estratégias metacognitivas; aprendizagem.
\end{abstract}

\section{Metacognition: A Support to the Learning Process}

\begin{abstract}
Based on literature review, this article aims to contribute to the clarifaction of the concept of metacognition. Therefore, it tries to highlight the role of metacognitive strategies to the development of effective ways of dealing with information from the enviroment and with their own thinking processes. It is argued that although there is a controversy over the concept of metacognition, it is unquestionable that it contributes to the learning processes since the training which involves metacognitive activities resulted in better school achievements.

Keywords: Metacognition; metacognitive strategies; learning.
\end{abstract}

Durante algumas décadas, as investigações no âmbito da aprendizagem centraram-se nas capacidades cognitivas e nos fatores motivacionais como os dois determinantes principais da realização escolar. A partir da década de 1970, uma terceira categoria de variáveis tem sido extensivamente estudada, a dos processos metacognitivos que coordenam as aptidões cognitivas envolvidas na memória, leitura, compreensão de textos, etc. (Brown, 1978; Flavell \& Wellman, 1977; Weinert \& Kluwe, 1987). Por exemplo, Pressley (1986), no seu modelo de Bom Utilizador de Estratégias - "Good Strategy User", realça que, em termos de realização escolar, para além da utilização de estratégias, é importante o conhecimento sobre quando e como utilizá-las, sobre a sua utilidade, eficácia e oportunidade. A este conhecimento, bem como à faculdade de planificar, de dirigir a compreensão e de avaliar o que foi aprendido, Flavell atribuiu a designação de metacognição ${ }^{2}$.

A falta de êxito de alguns estudos com o intuito de promover a utilização de estratégias e/ou modificações, nas já utilizadas (Flavell \& Wellman, 1977), e a verificação de diferenças significativas no desempenho escolar observadas, não apenas em função da utilização de estratégias cognitivas, mas também de estratégias metacognitivas, levou alguns autores a concluírem que os bons alunos são mais aptos

1 Endereço para correspondência: Urb. Vilabeira, Lote 10, $5^{\circ}$ direito, 3500 733, Viseu, Portugal. Fone: 232426520. E-mail: celia.ribeiro@mail.telepac.pt 2 Etimologicamente, a palavra metacognição significa para além da cognição, isto é, a faculdade de conhecer o próprio ato de conhecer, ou, por outras palavras, consciencializar, analisar e avaliar como se conhece. tanto na utilização de estratégias para adquirir, organizar e utilizar o seu conhecimento, como na regulação do seu progresso cognitivo ${ }^{3}$.

Deste modo, pode dizer-se que o interesse em torno desta temática advém, por um lado, da identificação de um papel determinante na eficácia de certos comportamentos. Por exemplo, observou-se que os sujeitos eficientes na execução de tarefas acadêmicas possuíam também competências metacognitivas bem desenvolvidas, pois demonstraram compreender a finalidade da tarefa, planificar a sua realização, aplicar e alterar conscientemente estratégias

3 Apesar do termo metacognição ser relativamente recente na literatura entrou em voga por volta dos anos 1970, sendo introduzido na Psicologia por Flavell —, já desde o início do século que pedagogos e psicólogos (Dewey, 1910; Huey, 1908, 1968; Thorndike, 1917, citados em Brown, 1987) demonstraram estar conscientes de que o estudo e a leitura envolvem um tipo de atividades agora denominadas de metacognitivas.

Por exemplo, podemos encontrar nas idéias de Thorndike aspectos relacionados com o que é actualmente considerado metacognição. Segundo este autor, ler um parágrafo é resolver um problema, pois consiste na selecção dos elementos certos da situação e a sua colocação nas relações certas. O sujeito deve levar a cabo determinadas atividades, como por exemplo: seleccionar, dominar, enfatizar, correlacionar e organizar, sob a orientação de um objetivo ou exigência/requisito.

Cavanaugh e Perlmutter (1982) referem ainda o trabalho de Baldwin (1909), autor que utilizava os questionários introspectivos para examinar as estratégias de estudo, como o precursor do auto conhecimento dos processos de pensamento e resultados das realizações.

Contudo, apesar do contributo de muitos teóricos e investigadores para a compreensão da metacognição e da sua relação com a aprendizagem (Lefebvre-Pinard \& Pinard, 1985; Pressley, 1986), ocupam lugar de destaque, nesta área, os trabalhos desenvolvidos por John Flavell, Ann Brown e colaboradores. 
de estudo e avaliar o seu próprio processo de execução (Flavell \& Wellman, 1977). Por outro, foi também demonstrado que a metacognição exerce influência em áreas fundamentais da aprendizagem escolar, tais como, na comunicação e compreensão oral e escrita e na resolução de problemas, constituindo assim, um elemento chave no processo de "aprender a aprender" (Valente, Salema, Morais \& Cruz, 1989).

Segundo Brown (1978), o reconhecer a dificuldade na compreensão de uma tarefa, ou tornar-se consciente de que não se compreendeu algo, é uma habilidade que parece distinguir os bons dos maus leitores. Os primeiros sabem avaliar as suas dificuldades e/ou ausências de conhecimento, o que lhes permite, nomeadamente, superá-las, recorrendo, muitas vezes, a inferências feitas a partir daquilo que sabem. Esta autora chama, assim, a atenção para a importância do conhecimento, não só sobre aquilo que se sabe, mas também, sobre aquilo que não se sabe, evitando assim, o que designa de ignorância secundária - não saber que não se sabe.

A metacognição pode ainda exercer influência sobre a motivação (Jones, 1988), pois o fato dos alunos poderem controlar e gerir os próprios processos cognitivos lhes dá a noção da responsabilidade pelo seu desempenho escolar e gera confiança nas suas próprias capacidades (Morais \& Valente, 1991).

Assim, é suposto que a prática da metacognição conduz a uma melhoria da atividade cognitiva e motivacional e, portanto, a uma potencialização do processo de aprender. Isto é, o conhecimento que o aluno possui sobre o que sabe e o que desconhece acerca do seu conhecimento e dos seus processos, parece ser fundamental, por um lado, para o entendimento da utilização de estratégias de estudo pois, presume-se que tal conhecimento auxilia o sujeito a decidir quando e que estratégias utilizar e, por outro, ou consequentemente, para a melhoria do desempenho escolar.

\section{Esclarecimento da Noção de Metacognição}

Apesar de ser reconhecida a importância da metacognição no processo de aprendizagem, parece não existir ainda uma definição unívoca. Uma breve incursão pela literatura levanos, de imediato, a refletir sobre dois aspectos: por um lado, a dificuldade em estabelecer a distinção entre o que é meta e o que é cognitivo e, por outro, parece gerar alguma confusão a utilização de um termo singular para um problema multifacetado (Brown, 1987) ${ }^{4}$.

\footnotetext{
${ }^{4}$ Um domínio onde este problema parece estar acentuado é no da leitura e, por conseguinte, do estudo. De acordo com Flavell (1976), o auto-questionamento sobre um texto pode funcionar não apenas para aumentar o seu conhecimento (função cognitiva), mas também para o monitorizar (função metacognitiva). Esta afirmação demonstra a inter-relação das funções cognitivas e metacognitivas, isto é, uma determinada atividade pode ser vista como uma estratégia (olhar para os pontos principais), possuir uma função de monitorização (uma atividade metacognitiva), e ser uma reflexão sobre o conhecimento (também uma atividade metacognitiva) (Brown, 1987).
}

No que concerne ao primeiro aspecto, no nível superficial, parece fácil estabelecer a distinção: enquanto a cognição, em termos restritos, se refere a um tipo específico de representação dos objetos e fatos (isto é, representações proposicionais) e, num sentido lato, a qualquer tipo de representação da informação proveniente do meio, incluindo todos os tipos de representações multidimensionais (Ex.: imagens espaciais) (Kuhl \& Kraska, 1989). A metacognição diz respeito, entre outras coisas, ao conhecimento do próprio conhecimento, à avaliação, à regulação e à organização dos próprios processos cognitivos. De acordo com Weinert (1987), as metacognições podem ser consideradas cognições de segunda ordem: pensamentos sobre pensamentos, conhecimentos sobre conhecimentos, reflexões sobre ações.

A outra fonte de confusão em torno da metacognição relaciona-se com a amplitude da utilização do termo na literatura psicológica. Perspetivada por diferentes correntes teóricas, tem provocado acesa polêmica concetual e múltiplas orientações de investigação. Por exemplo, enquanto Campione, Brown e Ferrara (1982, citados em Neimark, De Lisi \& Newman, 1985) e Flavell (1981a, 1981b) se referem à metacognição, quer como o conhecimento do próprio conhecimento, dos próprios processos cognitivos e das suas formas de operação, quer como controle executivo (que envolve a regulação ou monitorização cognitiva). Cavanaugh e Perlmutter (1982), na sua revisão sobre a metamemória ${ }^{5}$, realçam somente o conhecimento que o sujeito possui acerca dos seus processos cognitivos, excluindo os processos executivos. De referir também Lefebvre-Pinard e Pinard (1985) que enfatizam somente o controle executivo ${ }^{6}$.

Assim, como objeto de investigação e no domínio educacional encontramos duas formas essenciais de entendimento da metacognição: conhecimento sobre o conhecimento (tomada de consciência dos processos e das competências necessárias para a realização da tarefa) e controle ou auto-regulação (capacidade para avaliar a execução da tarefa e fazer correções quando necessário - controle da atividade cognitiva, da responsabilidade dos processos executivos centrais que avaliam e orientam as operações cognitivas) ${ }^{7}$.

\footnotetext{
${ }^{5}$ Este termo é normalmente utilizado para referir o conhecimento sobre a memória, e não, o seu controle (Sternberg, 1979).

6 Vygotsky (1978) foi um dos primeiros investigadores no âmbito da psicologia cognitiva, a postular a relação direta entre a consciência dos próprios processos cognitivos e a capacidade de controlá-los.

${ }^{7}$ Inicialmente, os autores usaram o termo meta para se referirem apenas à consciência reflexiva dos processos cognitivos. No entanto, outros autores foram mais além (Brown, 1978; Flavell, 1979) e incluíram também o controle da cognição na definição de metacognição. Ao mesmo tempo, investigadores no campo da deficiência mental propuseram a existência de uma série de processos responsáveis pelo controle da cognição, que se tornaram objeto de estudo (Butterfield, Wambold \& Belmont, 1973, citados em Brown, Bransford, Ferrara \& Campione, 1983) e foram designados de processos executivos. Estes e respectivas funções foram incluídos nas definições apresentadas por Flavell e Brown de metacognição.
} 
Embora possuam diferentes fontes e diferentes problemas, o conhecimento e a regulação da cognição encontram-se intimamente relacionados. Tentativas para separá-los levariam a uma grande simplificação (Brown, 1987); por isso, consideraremos as duas dimensões da metacognição, que passaremos a explicitar.

Como já referimos, foi nos anos 1970 que Flavell e colaboradores começaram a desenvolver estudos relacionados com a metacognição, nomeadamente com a metamemória. Com base nestes estudos, Flavell e Wellman (1977) sugeriram que o conhecimento metacognitivo se desenvolve através da consciencialização, por parte do sujeito, sobre o modo como determinadas variáveis interagem no sentido de influenciar os resultados das atividades cognitivas.

Assim, numa primeira tentativa de clarificação deste conceito, Flavell e Wellman (1977) elaboraram um sistema para o desenvolvimento da metamemória, que inclui dois componentes: a sensibilidade o conhecimento das variáveis dapessoa, da tarefa e da estratégia. Segundo estes autores, para que a memorização ou a recordação se tornem possíveis, um sujeito deve aprender a identificar em que situações há necessidade de recorrer a determinadas ações ou estratégias (sensibilidade) e desenvolver o conhecimento sobre a influência das variáveis da pessoa, da tarefa e da estratégia.

A variável pessoa envolve três categorias de conhecimento: intraindividual, interindividual e universal. A primeira refere-se ao conhecimento sobre si próprio áreas fortes e fracas, interesses, atitudes, etc; a segunda referese ao conhecimento sobre as diferenças entre si próprio e os outros; e a terceira refere-se a todo o conhecimento dominante numa cultura que veicula certas idéias acerca da aprendizagem, como, por exemplo, que a memória tem uma capacidade limitada.

A variável tarefa refere-se ao conhecimento sobre a natureza da informação com que um sujeito é confrontado (escassa ou abundante, imprecisa ou rigorosa) e sobre os critérios da tarefa a realizar. A informação ou o material a aprender varia em função da sua familiaridade e da forma como é apresentado, devendo o sujeito adaptar as suas respostas a essas características (Ex.: o conhecimento prévio dos objetivos da leitura de um texto orientará a forma, mais ou menos exigente, como a informação será "trabalhada"). Em outras palavras, diferentes tipos de tarefas exigem diferentes tipos de processamento da informação.

A variável estratégia inclui informações sobre os meios, processos ou ações que permitem ao sujeito atingir os objetivos com maior eficácia numa determinada tarefa. Neste sentido, possuir um reportório razoável de estratégias não é o suficiente, é também necessário ter um conhecimento aprofundado sobre elas.

Ainda, que as variáveis da pessoa, da tarefa e da estratégia interagem entre si, fazendo, por isso, sentido que o aprendiz X, mas não o aprendiz $\mathrm{Y}$, utilize a estratégia A mais do que a estratégia $\mathrm{B}$, porque a tarefa é mais deste tipo do que do outro.

Posteriormente, Flavell (1979) desenvolve um modelo global de monitorização cognitiva que inclui quatro aspectos inter-relacionados: 1) conhecimento metacognitivo (que aglutina os componentes sensibilidade e conhecimento das variáveis da pessoa, da tarefa e da estratégia); 2) experiências metacognitivas; 3) objetivos; e, 4) açoes (ou estratégias).

O conbecimento metacognitivo é definido como o conhecimento ou crença que o aprendiz possui sobre si próprio, sobre os fatores ou variáveis da pessoa, da tarefa, e da estratégia e sobre o modo como afetam o resultado dos procedimentos cognitivos. Contribui para o controle das condutas de resolução, permitindo ao aprendiz reconhecer e representar as situações, ter mais fácil acesso ao reportório das estratégias disponíveis e selecionar as suscetíveis de se poderem aplicar. Permite, também, avaliar os resultados finais e/ou intermédios e reforçar a estratégia escolhida ou de a alterar, em função da feitura de avaliações.

As experiências metacognitivas prendem-se com o foro afetivo e consistem em impressões ou percepções conscientes que podem ocorrer antes, durante ou após a realização de uma tarefa. Geralmente, relacionam-se com a perceção do grau de sucesso que se está a ter e ocorrem em situações que estimulam o pensar cuidadoso e altamente consciente, fornecendo oportunidades para pensamentos e sentimentos acerca do próprio pensamento.

Deste modo, podemos falar em experiência metacognitiva, sempre que é experienciada uma dificuldade, uma falta de compreensão, um sentimento de que algo está a correr mal (Ex.: se alguém tem subitamente o sentimento de ansiedade, porque não está a compreender algo, mas que necessita e quer compreender, este sentimento poderia ser denominado de experiência metacognitiva). Estas experiências são importantes, pois é, sobretudo, através delas que o aprendiz pode avaliar as suas dificuldades e, consequentemente, desenvolver meios de as superar.

Podemos assim considerar, tal como Flavell (1987), que o conhecimento metacognitivo e as experiências metacognitivas estão interligados, na medida que o conhecimento permite interpretar as experiências e agir sobre elas. Estas, por sua vez, contribuem para o desenvolvimento e a modificação desse conhecimento.

O terceiro aspecto diz respeito aos objetivos, implícitos ou explícitos, que impulsionam e mantêm o empreendimento cognitivo e que podem ser impostos pelo 
professor ou selecionados pelo próprio aprendiz. De referir que o objetivo colocado por este pode ser diferente do "imposto" pelo professor, podendo modificar-se no decorrer da realização da tarefa.

As ações que, pelo seu significado, tratam-se dos conceitos que mais nos interessam abordar, correspondem às estratégias utilizadas para potencializar e avaliar o progresso cognitivo. Estas podem ser de dois tipos: se forem utilizadas a serviço do progresso da monitorização, ou seja, sempre que está em causa a avaliação da situação, as ações podem ser entendidas como estratégias metacognitivas, produzindo experiências metacognitivas e resultados cognitivos. Porém, se forem utilizadas para produzir progresso cognitivo, ou seja, quando a finalidade consiste em atingir o objetivo cognitivo, podem ser entendidas como estratégias cognitivas, produzindo igualmente experiências metacognitivas e resultados cognitivos. Como estes procedimentos podem ser aplicados a serviço da compreensão (utilizados para compreender e/ou avaliar a compreensão) depende do conhecimento metacognitivo, das experiências metacognitivas e do modo como o critério da tarefa é entendido (Brown, Campione \& Day, 1981). As estratégias cognitivas podem surgir na seqüência da ação das estratégias metacognitivas, quando, face a uma avaliação da situação, o aprendiz conclui pela necessidade de utilização de novas estratégias.

Flavell (1987) refere que, enquanto as estratégias cognitivas são destinadas simplesmente a levar o sujeito a um objetivo cognitivo, as estratégias metacognitivas propõem-se avaliar a eficácia das primeiras. Por exemplo, algumas vezes procedemos a uma leitura lenta simplesmente para aprender o conteúdo (estratégia cognitiva); outras vezes, lemos rapidamente para ter uma idéia acerca da dificuldade ou facilidade da aprendizagem do seu conteúdo (estratégia metacognitiva). Deste modo, aprendemos sobre as estratégias cognitivas para fazermos progressos cognitivos e sobre as estratégias metacognitivas para monitorizar o progresso cognitivo. Para este autor, a utilização de estratégias metacognitivas é, geralmente, operacionalizada como a monitorização da compreensão, que requer o estabelecimento de objetivos de aprendizagem, a avaliação do grau em que estão a ser alcançados e, se necessário, a modificação das estratégias que têm sido utilizadas para os alcançar.

De acordo com as classificações apresentadas, podemos identificar três aspectos envolvidos no construto metacognição: 1) o conhecimento dos processos cognitivos e do produto desses processos; 2) o conhecimento das propriedades pertinentes relativas à aprendizagem da informação ou dos dados (Ex.: saber que a estrutura da informação suscita uma memorização mais eficaz); e 3) a regulação dos processos cognitivos.
Para Brown (1987), a metacognição encontra-se também associada, por um lado, ao conhecimento sobre os próprios recursos cognitivos ou sobre as estratégias mais apropriadas para a realização de uma tarefa específica (conhecimento do conhecimento) e, por outro, à regulação do conhecimento. Esta envolve a utilização de mecanismos auto-regulatórios durante a realização de uma tarefa, que incluem: a planificação, a verificação, a monitorização, a revisão e a avaliação das realizações cognitivas.

Brown (1987), apesar de assumir estas duas dimensões, acrescenta que as mesmas se podem distinguir, pois, enquanto o conhecimento é estável, passível de verbalização e falível, o controle é instável e nem sempre passível de verbalização. Por outro lado, o controle é dependente da situação e da tarefa e, somente quando o sujeito regula ou monitoriza as atividades cognitivas é que pode se beneficiar dos fracassos, conseguindo abandonar as estratégias inapropriadas.

Importa, também, analisar como o conhecimento metacognitivo se encontra relacionado com os processos cognitivos. Na perspectiva de Lawson (1984), o ato de refletir envolve a avaliação do curso/fluxo da cognição, que inclui a análise do progresso em termos de planos, monitorização e modificação da cognição. Isto sugere que nos tornamos "conscientes da nossa consciência" e faz da cognição o objeto da nossa cognição através da operação dos processos executivos (ver Figura 1).
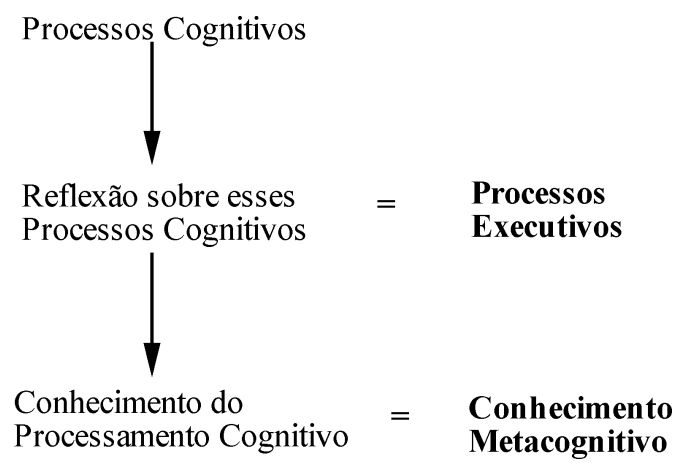

Figura 1. Conhecimento metacognitivo como o resultado dos processos executivos (adaptado de Lawson, 1984).

Assim, de acordo com o referido autor, a designação metacognição deve ser aplicada ao conhecimento metacognitivo, visto como distinto e resultante da operação dos processos executivos, que, por sua vez, estão relacionados com o controle da cognição. A aplicação deste conceito à aprendizagem sugere que a aquisição do conhecimento 
metacognitivo deve ser vista como um processo controlado, que requer uma constante e consciente monitorização.

Um outro ponto mais ou menos esclarecido no âmbito desta temática é de que o conhecimento metacognitivo requer um envolvimento ativo do aprendiz na aprendizagem. De acordo com a maioria dos autores (Jacobs \& Paris, 1987; Lawson, 1984), requer a reflexão consciente sobre os atributos cognitivos pessoais, estilo cognitivo, estratégias e conhecimento dos esquemas (Figura 2).

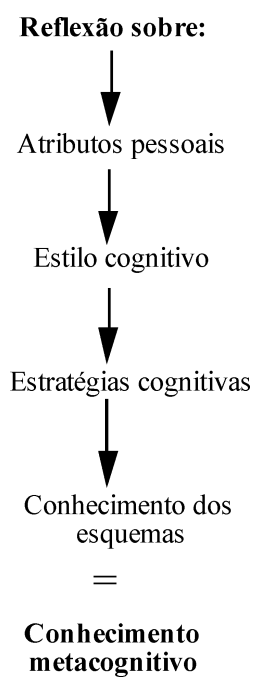

Figura 2. Conhecimento metacognitivo como resultado da reflexão sobre o self (adaptado de Lawson, 1984).

$\mathrm{Na}$ literatura, um outro ponto de discussão em torno desta temática prende-se com a consciência ou não dos aspectos metacognitivos. Embora o conhecimento metacognitivo tenha sido definido como consciente e passível de ser relatado, na opinião de Lefebvre-Pinard (1983), o fato do sujeito exercer um controle sobre o seu pensamento e conduta não equivale a afirmar que se trata sempre de uma atividade consciente e deliberada. Também Flavell (1981a, 1981b), que inicialmente caracteriza as experiências metacognitivas como conscientes, mais tarde (Flavell, 1985) considera que as mesmas podem incluir aspectos não conscientes.

Jacobs e Paris (1987), por outro lado, referem que só se pode falar de metacognição quando se trata de conhecimento e controle consciente: "o conhecimento acerca da cognição pode ser demonstrado, comunicado, examinado e discutido" (p. 258). Deste modo, a atividade cognitiva não consciente, como, por exemplo, as aptidões automáticas, não deve ser incluídas no domínio da metacognição. Weinert (1987) defende que a metacognição abrange não só a tomada de consciência dos processos cognitivos, mas também o controle deliberado e consciente dos mesmos, e refere como atributos do pensamento metacognitivo: 1) o conhecimento sobre os próprios processos cognitivos; 2) a tomada de consciência desses processos; e 3) o seu controle.

Assim, enquanto alguns autores (Flavell, 1985) realçam a possibilidade de um conhecimento e um controle metacognitivos inconscientes, outros (Jacobs \& Paris, 1987; Lefebvre-Pinard, 1983) salientam o carácter consciente da regulação das condutas e do pensamento.

Inserido ainda no processo de clarificação do conceito metacognição, de destacando a contribuição de Paris, Lipson e Wixson (1983) que discriminam, na metacognição, três tipos de conhecimento: 1) declarativo (consciência do que se sabe e das estratégias ao serem utilizadas); 2) executivo (conhecimento de como realizar determinada tarefa ou aplicar uma estratégia); 3) condicional (conhecimento de quando e onde usar uma estratégia particular). A aquisição destes conhecimentos procede em termos graduais, isto é, num primeiro nível, somente declarativo e, posteriormente, declarativo, executivo e condicional, simultaneamente.

Uma outra questão pertinente prende-se à origem da metacognição no desenvolvimento. Tem sido verificado que as crianças são bastante limitadas no seu conhecimento e na consciência dos fenômenos cognitivos (Flavell, 1981b) e não percebem os benefícios da utilização de estratégias na execução das tarefas (Paris \& Lindauer, 1982). Por esta razão, alguns autores pensam que a metacognição é um atributo que se desenvolve tardiamente. Contudo, não há consenso acerca do momento exato do seu aparecimento.

Por exemplo, enquanto para Garner e Alexander (1989) as crianças mais novas, além de apresentarem limitações no nível da quantidade de conhecimento que possuem, manifestam-se menos capazes de refletir sobre a forma como o organizam. Só por volta dos 11 anos de idade se verifica o desabrochar desse atributo do pensamento formal. Para Costa (1984), o conhecimento metacognitivo emerge mais cedo, por volta dos 7 anos de idade, mas tal como outros tipos de conhecimento, é adquirido de uma forma lenta e gradual, tendo um aumento considerável na préadolescência e adolescência.

Quanto aos fatores que influenciam o seu desenvolvimento, é suposto que as formas mais primitivas de conhecimento metacognitivo são, em primeira instância, determinadas pelo meio familiar. À medida que a criança prossegue na escola, é provável que a atividade metacognitiva seja um produto do estilo de ensino dos professores em conjunto com as experiências individualizadas numa variedade de contextos de aprendizagem (Kurtz \& Borkowski, 1987). Depreende- 
se assim a importância dos fatores externos para o desenvolvimento da metacognição.

Nesta linha de pensamento, encontramos Vygotsky (1978), que refere que as situações sociais, nas quais a criança interage com peritos num domínio de resolução de problemas, têm um contributo importante para a aprendizagem. Segundo este autor, o processo fundamental do desenvolvimento é a internalização gradual e a personalização do que foi originalmente uma atividade social. Ou seja, inicialmente é o adulto (pais, professores, etc.) que controla e guia a atividade da criança; gradualmente o adulto e a criança partilham as funções de resolução do problema, em que a criança toma a iniciativa e o adulto a corrige quando falha; finalmente, a criança assume o controle da própria atividade (Brown, 1987).

Assim, o desenvolvimento de aptidões metacognitivas realiza-se normalmente através da internalização gradual de aptidões regulatórias, que são primeiramente experienciados pela criança em situações sociais. Após repetidas experiências com peritos (pais, professores, etc.), que criticam, avaliam e ampliam os limites das suas experiências, a criança desenvolve aptidões de auto-regulação.

Flavell (1987), partilhando, de certo modo, desta opinião, acrescenta que o conhecimento metacognitivo e a experiência metacognitiva se desenvolvem à medida em que ocorre o desenvolvimento cognitivo, o qual vai possibilitar o aparecimento de novas operações cognitivas. Por um lado, estas operações são necessárias para a aquisição de conhecimentos metacognitivos e facilitam a maturação de outros já existentes e, por outro, criam condições para novas experiências metacognitivas. Por exemplo, o pensamento hipotético-dedutivo que surge na adolescência abre novas alternativas às possibilidades de planejamento de uma atividade, permitindo a consideração de diferentes meios para atingir um objetivo. À medida em que o aprendiz vai empreendendo determinadas aprendizagens escolares, vai sendo capaz de dominar melhor certas tarefas. Este controle lhe facilita a ocorrência de experiências metacognitivas que, por sua vez, vão possibilitar a tomada de consciência das dificuldades encontradas na realização daquelas tarefas e dos meios para superá-las (Silva \& Sá, 1993).

Por fim, é de referir duas mudanças no desenvolvimento que, possivelmente, contribuem para a aquisição da metacognição: 1) o desenvolvimento do sentido do self, como um agente cognitivo e como o centro causal da própria atividade cognitiva (ou locus de controle interno), que promoveria a monitorização e a regulação das próprias atividades cognitivas e 2) o aumento na capacidade de planejamento. Segundo Flavell (1987), em termos gerais, o sujeito que representa e inter-relaciona ações passadas, presentes e futuras estaria numa melhor posição para fazer progressos metacognitivos, comparativamente a outros.

\section{Metacognição e Aprendizagem}

Apesar de toda a polêmica existente à volta deste conceito, tem sido observada a sua contribuição para a potencialização da aprendizagem. Os treinos que contemplam, além de atividades cognitivas, atividades metacognitivas, têm originado melhores resultados em termos de realização escolar.

Segundo Paris e Winograd (1990), a metacognição, no que diz respeito especificamente à aprendizagem, pode assumir dois significados: a avaliação de recursos e a metacognição em ação. A avaliação de recursos ou auto-apreciação cognitiva refere-se a reflexões pessoais sobre o estado dos conhecimentos e competências cognitivas, sobre as características da tarefa que influenciam a dificuldade cognitiva e sobre as estratégias disponíveis para a realização da tarefa. A metacognição em ação ou auto-controle cognitivo diz respeito a reflexões pessoais sobre a organização e planificação da ação - antes do início da tarefa, nos ajustamentos que se fazem enquanto se realiza a tarefa e nas revisões necessárias à verificação dos resultados obtidos.

Idealmente, os professores funcionam como mediadores na aprendizagem e agem como promotores da autoregulação ao possibilitarem a emergência de planos pessoais. De acordo com Brown (1987), estes assumem um papel fundamental na preparação dos alunos para planejar e monitorar as suas próprias atividades.

De modo a estimular a metacognição, o professor tem toda a vantagem em multiplicar as situações abertas de investigação, as resoluções de problemas complexos no decurso dos quais o sujeito é levado a escolher entre várias alternativas e a antecipar as conseqüências destas escolhas. Só este gênero de atividade pode dar ao aluno, sobretudo se tem dificuldades, oportunidade de conduzir de maneira refletida as suas próprias operações cognitivas (Grangeat, 1999).

Torna-se assim necessário que a escola não se circunscreva a ser, apenas, um espaço de difusão dos saberes e se defina, antes:

como um contexto que estimule os seus alunos a apropriar-se e a construir, de uma forma progressiva, o seu património pessoal de metaconhecimentos, ou seja, de conhecimentos sobre o modo como se adquire, gere, utiliza e alarga o seu campo de saberes. (Cosme \& Trindade, 2001, p. 13)

Em sintese, a consideração da aprendizagem numa orientação metacognitiva apresenta diversas vantagens, de entre as quais a de salientar: 1) a auto-apreciação e o auto-controle cognitivos como formas de pensamento 
que o sujeito pode desenvolver e que lhe permitem ter um papel ativo e construtivo no seu próprio conhecimento o foco de atuação, no nível metacognitivo, é desenvolver nos alunos aquelas competências, tanto quanto o seu desenvolvimento e prontidão cognitivos lhe permitem; 2) a metacognição abre novas perspectivas para o estudo das diferenças individuais no rendimento escolar, uma vez que destaca o papel pessoal na avaliação e controle cognitivos — alunos com idênticas capacidades intelectuais podem ter diferentes níveis de realização escolar, devido à forma como cada um atua sobre os seus próprios processos de aprendizagem; 3) a metacognição, apesar de estar dependente do desenvolvimento cognitivo, como já foi referido, também favorece e é o motor do próprio desenvolvimento, uma vez que permite ao sujeito ir mais longe no seu nível de realização.

Deste modo, a eficácia da aprendizagem não é dependente apenas da idade, experiência e nível intelectual, mas também da aquisição de estratégias cognitivas e metacognitivas que possibilitem ao aluno planejar e monitorar o seu desempenho escolar; isto é, que permitem a tomada de consciência dos processos que utiliza para aprender e a tomada de decisões apropriadas sobre que estratégias utilizar em cada tarefa e, ainda, avaliar a sua eficácia, alterando-as quando não produzem os resultados desejados (Silva \& Sá, 1993).

Nesta perspectiva, para aprender é preciso aprender como fazer para aprender, que não basta fazer e saber, mas é preciso saber como se faz para saber e como se faz para fazer (Grangeat, 1999). A metacognição pode, então, ser vista como a capacidade chave de que depende a aprendizagem, certamente a mais importante: aprender a aprender, o que por vezes não tem sido contemplado pela escola.

\section{Referências}

Brown, A. L. (1978). Knowing when, where, and how to remember: A problem of metacognition. Em R. Glaser (Org.), Advances in instructional psychology (Vol. 1, pp. 77-165). Hillsdale, N.J.: Erlbaum.

Brown, A. L. (1987). Metacognition, executive control, self-regulation, and other more mysterious mechanisms. Em F. E. Weinert \& R. Kluwe (Orgs.), Metacognition, motivation, and understanding (pp. 1-16). Hillsdale, N. J.: Erlbaum.

Brown, A. L., Campione, J. C. \& Day, J. D. (1981). Learning to learn: On training students to learn from the texts. Educational Researcher, 10, 1421.

Brown, A. L., Bransford, J. D., Ferrara, R. A. \& Campione, J. C. (1983). Learning, remembering, and Understanding. Em P. H. Mussen, J. H. Flavell \& E. M. Markman (Orgs.), Handbook of cbild psychology cognitive Development ( $4^{a}$ ed.) (Vol. 3, pp. 77-166). New York: John Wiley \& Sons.

Cavanaugh, J. C. \& Perlmutter, M. (1982). Metamemory: A critical examination. Child Development, 53, 11-28.
Cosme, A. \& Trindade, R. (2001). Área de estudo acompanhado. O essencial para ensinar e aprender. Porto: Edições Asa.

Costa, A. L. (1984). Mediating the metacognitive. Educational Leadership, 42(3), 57-62.

Di Vesta, F. (1987). The cognitive movement and education. Em J. A. Glover \& R. R. Ronning (Orgs.), Historical foundations of educational psychology. New York: Plenum Press.

Flavell, J. H. (1976). Metacognitive aspects of problem solving. Em L. B. Resnick (Orgs.), The nature of intelligence (pp. 231-235). Hillsdale, N.Y.: Erlbaum.

Flavell, J. H. (1979). Metacognition and cognitive monitoring: A new area of cognitve-developmental inquiry. American Psychologist, 34(10), 906911.

Flavell, J. H. (1981a). Cognitive monitoring. Em W. P. Dickson (Orgs.), Children's oral communication skills (pp. 35-60). New York: Academic Press.

Flavell, J. H. (1981b). Metacognition and cognitive monitoring: A new area of cognitive-developmental inquiry. Em H. Parke (Orgs.), Contemporary readings in child psychology (pp. 165-169). New York: McGraw Hill.

Flavell, J. H. (1985). Developpment métacognitif. Em J. Bideaud \& M. Richelle (Orgs.), Psychologie développmentale: Problémes et réalités (pp. 29-41). Bruxelles: Pierre Mardaga.

Flavell, J. H. (1987). Speculations about the nature and development of metacognition. Em F. E. Weinert \& R. Kluwe (Orgs.), Metacognition, motivation, and understanding (pp. 1-16). Hillsdale, N. J: Erlbaum.

Flavell, J. H. \& Wellman, H. M. (1977). Metamemory. Em R. V. Kail \& J. W. Hagen (Orgs.), Perspetives on the development of memory and cognition (pp. 333). Hillsdale, N.J.: Erlbaum.

Garner, R. \& Alexander, P. A. (1989). Metacognition: Answered and unanswered questions. Educational Psychologist, 24(2), 145-158.

Grangeat, M. (1999). A metacognição, um apoio ao trabalho dos alunos. Porto: Porto.

Jacobs, J. E. \& Paris, S. G. (1987). Children's metacognition about reading : Issues in definition, measurement and instruction. Educational Psychologist, 22(3/4), 255-278.

Jones, B. F. (1988). Text learning strategy instruction:Guidelines from theory and practice. Em C. E. Weinstein, E. T. Goetz \& P. A. Alexander (Orgs.), Learning and study strategies: Issues in assessment, instruction, and evaluation (pp. 233-260). N. Y.: Academic Press.

Kuhl, J. \& Kraska, K. (1989). Self-regulation and metamotivation: Computational mechanisms, development, and assessment. Em R. Kanfer, P. Ackerman \& R. Cudeck (Orgs.), Abilities motivation methodology: The Minnesota symposium on learning and individual differences (pp. 343-374). N. J.: Erlbaum.

Kurtz, B. E. \& Borkowski, J. G. (1987). Development of strategic skills in impulsive and reflective children: A longitudinal study of metacognition. Journal of Experimental Child Psychology, 43(1), 129-148.

Lawson, M. J. (1984). Being executive about metacognition. Em J. R. Kirby (Org.), Cognitive strategies and educational performance (pp. 89-109). Orlando: Academic Press.

Lefebvre-Pinard, M. \& Pinard, A. (1985). Taking charge of one's cognitive activity: A moderator of competence. Em E. Neimark, R. de Lisi \& J. Newmam (Orgs.), Moderators of competence (pp. 191-212). Hillsdale, N.Y.: Erlbaum.

Morais, M. M. \& Valente, M. O. (1991). Pensar sobre o pensar: Ensino de estratégias metacognitivas para recuperação de alunos com dificuldades na compreensão da leitura na disciplina de língua portuguesa. Revista de Educação, 2(1), 35-56.

Neimark, E. D., De Lisi, R. \& Newman, J. L. (1985). Moderators of competence. Hillsdale, N. Y.: Erlbaum.

Paris, S. G. \& Lindauer, B. K. (1982). The development of cognitive skills during childwood. Em B. Wolman (Orgs.), Handbook of developmental psychology (pp. 333-349). Englewood Cliffs, N. J.: PrenticeHall.

Paris, S. G., Lipson, M. Y. \& Wixson, K. K. (1983). Becoming a strategic reader. Contemporary Educational Psychology, 8, 293-316. 
Paris, S. \& Winograd, P. (1990). How metacognition can promote academic learning and instruction. Em B. Jones \& L. Idol (Orgs.), Dimensions of thinking and cognitive instruction. Hillsdale, N. J.: Erlbaum.

Pressley, M. (1986). The relevance of good strategy user model to the teaching of mathematics. Educational Psychologist, 21, 139-161.

Silva, A. L. \& Sá, I. (1993). Saber estudar e estudar para saber. Colecção Ciências da Educação. Porto: Porto.

Sternberg, R. J. (1979). The nature of mental abilities. American Psychologist, 34(3), 214-230.

Valente, M. O., Salema, M. H., Morais, M. M. \& Cruz, M. N. (1989). A metacognição. Revista de Educação, 1(3), 47-51.

Vygotsky, L. S. (1978). Mind in society. Cambridge, Massachusetts: Harvard University Press.
Weinert, F. E. (1987). Metacognition and motivation as determinants of effective learning and understanding. Em F. E. Weinert \& R. Kluwe (Orgs.), Metacognition, motivation, and understanding (pp. 1-16), Hillsdale, N. J.: Erlbaum.

Weinert, F. E. \& Kluwe, R. H. (1987). Metacognition, motivation, and understanding. Hillsdale, N. J.: Erlbaum.

Sobre a autora

Célia Ribeiro é Psicóloga e Mestre em Psicologia com área de especialização em Psicologia Pedagógica

pela Faculdade de Psicologia e Ciências da Educação da Universidade de Coimbra.

Recebido: 08/04/2002

$1^{a}$ revisão: $03 / 07 / 2002$

Aceite final: $21 / 08 / 2002$ 